\title{
THE DISTANCES TO OPEN CLUSTERS FROM MAIN-SEQUENCE FITTING. I. NEW MODELS AND A COMPARISON WITH THE PROPERTIES OF THE HYADES ECLIPSING BINARY VB 22
}

\author{
Marc H. Pinsonneault and Donald M. Terndrup \\ Department of Astronomy, Ohio State University, Columbus, OH 43210; pinsono@astronomy.ohio-state.edu, \\ terndrup@astronomy.ohio-state.edu \\ RoBERT B. HANSON \\ University of California Observatories/Lick Observatory, Santa Cruz, CA 95064; hanson@ucolick.org \\ AND \\ JOHN R. STAUFFER \\ Infrared Processing and Analysis Center, California Institute of Technology, MS 100-22, \\ 770 South Wilson Avenue, Pasadena, CA 91125; stauffer@ipac.caltech.edu \\ Received 2003 June 2; accepted 2003 July 31
}

\begin{abstract}
In the first of a new series of papers on open cluster distances, we use updated stellar evolution models to construct an isochrone appropriate for the Hyades and compare it with the Hyades eclipsing binary system VB 22. We find that the absolute and relative luminosities of the two stars are in good agreement with the model but that the radii do not match the values inferred from eclipse data. We present evidence that there is a consistency problem with the flux ratios and the inferred radii and discuss possible theoretical effects that could be responsible for the mismatch in the radii. We derive a helium abundance for the Hyades of $Y=0.271 \pm 0.006$, which is equal within the errors to the Sun's initial helium abundance, even though the Hyades is considerably more metal-rich.
\end{abstract}

Subject headings: binaries: eclipsing — open clusters and associations: individual (Hyades) — stars: abundances — stars: distances

\section{INTRODUCTION}

The distances to Milky Way star clusters as derived from main-sequence fitting play a critical role in unraveling the history of the Galaxy and, via luminosity calibration of pulsating variables, in finding distances throughout the Local Group. Parallaxes from the Hipparcos satellite have provided precise measurements of the distances to the nearest open clusters, particularly the Hyades (Perryman et al. 1998), allowing stringent tests of the predictions of stellar evolutionary models (e.g., Lebreton, Fernandes, \& Lejeune 2001).

To go beyond the handful of clusters with trigonometric parallaxes (e.g., van Leeuwen 1999; Robichon et al. 1999; Makarov 2002) requires isochrones that are physically accurate and well calibrated over a wide range of temperature, ideally all along the main sequence. As is well known this is a nontrivial exercise, since the luminosity of the main sequence is a sensitive function of helium abundance and metallicity. Radii from stellar models depend on the treatment of convection, for which only simple phenomenological theories are available. Photometric colors and bolometric corrections are often poorly determined, especially for stars much hotter or cooler than the Sun, and are highly dependent on the details of model atmospheres employed in the computation of the isochrones. Rapidly rotating stars typically have large spots and chromospheric emission, not modeled in the computation of the isochrones, which could affect their colors especially in the blue and ultraviolet (van Leeuwen, Alphenaar, \& Meys 1987; Stauffer et al. 2003). As a result of some or all these effects, it is typically the case that even the best isochrones do not match the detailed shape of the main sequence as determined from photometry
(Terndrup et al. 2000; Castellani, Degl'Innocenti, \& Prada Moroni 2001; Lebreton et al. 2001).

Despite the complexity of the problem, it is now possible to determine more accurate absolute and relative distances to open clusters. Helioseismology has given us reliable measures of many parameters that directly or indirectly affect the radius, including the helium abundance, the amount of helium diffusion, and opacities in the convective zone (e.g., Bahcall, Pinsonneault, \& Basu 2001). Hipparcos parallaxes in the Hyades (Perryman et al. 1998; de Bruijne, Hoogerwerf, \& de Zeeuw 2001) and of nearby field stars (Jimenez et al. 2003; Percival, Salaris, \& Kilkenny 2003) can potentially provide the means of empirically correcting the isochrone color-temperature relation.

In addition, eclipsing binary stars provide a powerful test of the theory of stellar structure and evolution, particularly the mass/luminosity relation. This is especially true of systems in star clusters, where there are additional constraints on the age and abundances of the stars. As summarized by Lebreton et al. (2001), the Hyades cluster has five binaries where the components have measured masses. Of these systems only VB 22 has masses with a small enough uncertainty to place powerful constraints on the theoretical models; we therefore focus on VB 22 (=818 Tau, HD 27130). In this system, the relative magnitudes of VB 22A and VB 22B have been measured in several colors and the absolute radii have been inferred.

We use VB 22 as a test of both the absolute luminosities and effective temperatures of our models. We contend that the agreement in luminosity that we obtain justifies the construction of an empirical isochrone where the colors as a function of $M_{V}$ are adjusted to reproduce the morphology of the Hyades color-magnitude diagram. In addition, we 
show that the absolute magnitudes of the two components in the $B, V$, and $I$ bands provide support for the relative model luminosities and effective temperatures, even though the direct radii inferred from the binaries are not in agreement with the models. This step justifies holding the model effective temperatures fixed and varying the color calibrations when constructing the empirical isochrone, the details of which are discussed in the second paper in this series. Finally, we examine the question of the Hyades helium abundance and the ratio $\Delta Y / \Delta Z$ appropriate for chemical evolution of solar-neighborhood stars.

The VB 22 system has been studied extensively since McClure (1982) first used it to determine the distance to the Hyades and to constrain the mass-luminosity relationship. The most recent papers (Lastennet et al. 1999; Lebreton et al. 2001; Torres \& Ribas 2002) have yielded somewhat discordant results. Lebreton et al. (2001) claim evidence for a low helium abundance, while Lastennet et al. (1999) and Torres \& Ribas (2002) find that the luminosities of the models are consistent with the data if the Hipparcos distance is adopted. All authors note the apparent contradiction between the radii of theoretical models and those obtained from the eclipse data. In light of these results we believe that a careful analysis of the data and the theoretical models is warranted, with particular attention to the errors involved.

\section{AN ISOCHRONE FOR THE HYADES}

We begin with a new set of theoretical models described in detail by Sills, Pinsonneault, \& Terndrup (2000). The essential aspects of these models are repeated here for the reader's convenience.

We used the Yale Rotating Evolution Code (YREC) to construct evolutionary tracks over the mass range $0.25 \leq$ $\left(M / M_{\odot}\right) \leq 2.25$. YREC is a Henyey code that solves the equations of stellar structure in one dimension (Guenther et al. 1992) and that follows rotational evolution by treating the star as a set of nested, rotationally deformed shells. For this application, however, we used the code in its nonrotating mode; nonrotating stars of the age and masses considered here are structurally identical to those that are rotating (e.g., Sills et al. 2000), and solarlike Hyades stars are slow rotators (Radick et al. 1987; Paulson, Sneden, \& Cochran 2003). The chemical composition of each shell is updated separately using the nuclear reaction rates of Gruzinov \& Bahcall (1998). Composition changes due to microscopic diffusion can be calculated. The initial chemical mixture is the solar mixture of Grevesse \& Noels (1993), and for the Sun the models have a surface metallicity of $Z=0.0176$ at the age of the solar system. We use the latest OPAL opacities (Iglesias \& Rogers 1996) for the interior of the star down to temperatures of $\log T(\mathrm{~K})=4$. For lower temperatures, we use the molecular opacities of Alexander \& Ferguson (1994). For regions of the star with $\log T(\mathrm{~K}) \geq 6$, we used the OPAL equation of state (Rogers, Swenson, \& Iglesias 1996). For regions where $\log T(\mathrm{~K}) \leq 5.5$, we used the equation of state from Saumon, Chabrier, \& Van Horn (1995), which calculates particle densities for hydrogen and helium, including partial dissociation and ionization by both pressure and temperature. In the transition region between these two temperatures, both formulations are weighted with a ramp function and averaged. The equation of state includes both radiation pressure and electron degeneracy pressure. For the surface boundary condition, we experimented with several stellar atmosphere models as described below. For our base case, we adopted $Y=0.273$ for the Hyades and ignored diffusion (details to follow) and used the standard Böhm-Vitense mixing-length theory (Böhm-Vitense 1958; Cox \& Guili 1968) with $\alpha=1.72$ to match the solar radius $\left(R_{\odot}=6.9598 \times 10^{8} \mathrm{~m}\right)$ and luminosity $\left(L_{\odot}=3.8515 \times 10^{26} \mathrm{~W}\right)$ at the present age of the Sun (4.57 Gyr).

The evolutionary tracks were generated for a Hyades metallicity of $[\mathrm{Fe} / \mathrm{H}]=+0.13$ and scaled solar abundances (Boesgaard \& Friel 1990; Paulson et al. 2003). The tracks were interpolated to form an isochrone for an adopted age of $550 \mathrm{Myr}$, consistent with ages derived from models excluding convective overshoot (Perryman et al. 1998). Models with overshoot have ages $\approx 625 \mathrm{Myr}$, but since we are dealing with relatively low mass stars, the comparison with VB 22 is completely insensitive to the choice of cluster age. The color-temperature relation in Lejeune, Cuisinier, \& Buser (1998) was used to generate preliminary colors ${ }^{1}$ from the model parameters $M_{\mathrm{bol}}$ and $T_{\text {eff }}$. The resulting isochrone is given as Table 1; the sensitivity of the colors to the choice of color-temperature relation is discussed below. Note that this isochrone is not empirically calibrated to match the photometry of the Hyades main sequence, a necessary procedure discussed at length in our next paper.

The principal theoretical uncertainties in the models are the adopted mixture of heavy elements and the physics chosen for the solar calibration, in particular whether microscopic diffusion is included or not. In the Hyades, the average iron abundance $[\mathrm{Fe} / \mathrm{H}]$ is now determined to high precision $( \pm 0.01 \mathrm{dex})$, and the relative abundances are near solar for most of the elements that contribute significantly to the internal opacities (Paulson et al. 2003). The choice of model atmospheres and low-temperature opacities affects only the position in the H-R diagram for effective temperatures below $3500 \mathrm{~K}$; similar comments apply to the equation of state. The superb agreement between theory and data for helioseismology provides some real confidence in the accuracy of the ingredients of the models for stars similar to the Sun, such as VB 22.

Although the effects of microscopic diffusion do not matter for the Hyades themselves (the cluster is only $12 \%$ the age of the Sun), they make a difference for the choice of a calibrating solar model. The net effect of diffusion is a gradual decrease in the helium and heavy-element content in the outer layers, with a smaller fractional rise in the central values of these quantities. Compared to models with a uniform composition profile, solar models including diffusion have a higher overall helium abundance and require a higher value of $\alpha$ to match the solar radius and luminosity.

The proper thing to do, therefore, would be to calibrate the Hyades models using diffusion models for the Sun. For our base case (the isochrone in Table 1), however, we ignored this and instead used models that do not follow diffusion and that are calibrated using models of the homogeneous Sun, even though these are incompatible with seismology. The principal reason for this is that the effect of rotationally induced mixing, which diminishes the effects of diffusion, is difficult to model for stars much hotter than the Sun, where convection zones are very thin.

\footnotetext{
${ }^{1} V-I$ is on the Cousins system, while the $V-K_{s}$ colors use the (short) $K$ band.
} 
TABLE

Theoretical Isochrone FOR THE Hyades ( $Y=0.273$, $[\mathrm{FE} / \mathrm{H}]=+0.13)$

\begin{tabular}{|c|c|c|c|c|c|c|c|c|c|c|c|}
\hline$M_{V}$ & $T_{\text {eff }}$ & $M / M_{\odot}$ & $B-V$ & $V-I_{\mathrm{C}}$ & $V-K_{s}$ & $\begin{array}{l}10.65 \ldots \ldots \ldots \ldots \\
10.80 \ldots \ldots \ldots \ldots \ldots\end{array}$ & $\begin{array}{l}3555 \\
3535\end{array}$ & $\begin{array}{l}0.393 \\
0.376\end{array}$ & $\begin{array}{l}1.440 \\
1.443\end{array}$ & $\begin{array}{l}2.076 \\
2.107\end{array}$ & $\begin{array}{l}4.037 \\
4.082\end{array}$ \\
\hline $1.07 \ldots \ldots$ & 8279 & 2.252 & 0.112 & 0.110 & 0.316 & $10.95 \ldots \ldots \ldots \ldots \ldots$ & 3516 & 0.359 & 1.444 & 2.140 & 4.131 \\
\hline $1.20 \ldots \ldots \ldots \ldots \ldots$ & 8270 & 2.192 & 0.116 & 0.112 & 0.320 & 11.10 & 3498 & 0.342 & 1.443 & 2.173 & 4.186 \\
\hline $1.35 \ldots \ldots \ldots \ldots \ldots$ & 8236 & 2.127 & 0.126 & 0.122 & 0.333 & 11.25 & 3481 & 0.325 & 1.440 & 2.208 & 4.244 \\
\hline 1.50 . & 8158 & 2.062 & 0.143 & 0.141 & 0.360 & $11.40 \ldots \ldots \ldots \ldots$ & 3465 & 0.308 & 1.436 & 2.242 & 4.305 \\
\hline $1.65 \ldots \ldots \ldots \ldots \ldots$ & 8052 & 1.997 & 0.166 & 0.169 & 0.396 & $11.55 \ldots \ldots \ldots \ldots \ldots$ & 3450 & 0.292 & 1.431 & 2.276 & 4.364 \\
\hline $1.80 \ldots \ldots \ldots \ldots \ldots$ & 7942 & 1.934 & 0.191 & 0.200 & 0.436 & $11.70 \ldots \ldots \ldots \ldots \ldots$ & 3436 & 0.277 & 1.427 & 2.309 & 4.422 \\
\hline $1.95 \ldots \ldots \ldots \ldots \ldots$ & 7830 & 1.873 & 0.215 & 0.229 & 0.477 & $11.85 \ldots \ldots \ldots \ldots$ & 3421 & 0.261 & 1.422 & 2.342 & 4.479 \\
\hline $2.10 \ldots \ldots \ldots \ldots \ldots \ldots$ & 7706 & 1.815 & 0.241 & 0.254 & 0.520 & $11.96 \ldots \ldots \ldots \ldots \ldots$ & 3411 & 0.250 & 1.419 & 2.365 & 4.520 \\
\hline
\end{tabular}

For the base case, which we call the "no diffusion" models, we chose the helium abundance as illustrated in Figure 1 . We assumed that the helium abundance is a function of the heavy-element content $Z$ as given by $Y=Y_{p}+(\Delta Y /$ $\Delta Z) Z$, where $Y_{p}$ is the primordial helium abundance. We took $Y_{p}=0.245 \pm 0.002$, an intermediate value between estimates from cosmic nucleosynthesis and measures in metal-poor H II regions (see Bono et al. 2002; Thuan \& Izotov 2002 and references therein). The solar helium abundance in models lacking diffusion is $Y_{\odot}=0.266 \pm 0.001$; these require a mixing length set by $\alpha=1.74$. Adopting these values of $Y_{p}, Y_{\odot}$, and $\alpha$ yields $Y=0.273$ for the Hyades using the Sun's surface metal abundance of $Z_{\odot}=0.0176$

We also computed models using an alternative set of parameters calibrated on solar models that include diffusion

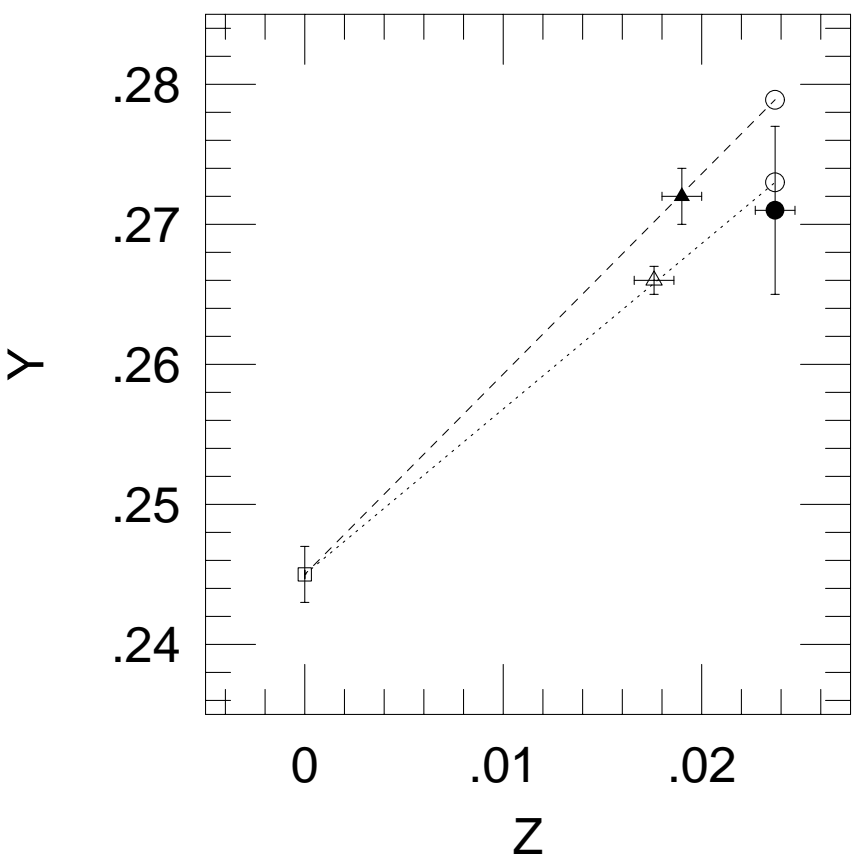

FIG. 1.-Derivation of the helium abundance $Y$ for the Hyades isochrone. The lower dashed line shows the extrapolation from the primordial helium abundance through models of the Sun that lack diffusion to the Hyades metal abundance $[\mathrm{Fe} / \mathrm{H}]=+0.13 \pm 0.01(Z=0.0237)$; this is the base case discussed in the paper and shown in Table 1. The upper line shows the extrapolation through solar models consistent with helioseismology, which would imply a higher helium abundance in the Hyades. Both initial values are shown as open circles. The filled circle shows the helium abundance for the Hyades found in this paper. 
TABLE 2

DisTances To VB 22

\begin{tabular}{ccl}
\hline \hline Reference & $\begin{array}{c}\text { Distance } \\
\text { Modulus }\end{array}$ & \multicolumn{1}{c}{ Method } \\
\hline Peterson \& Solensky 1988 ....... & $3.35 \pm 0.02$ & Orbital parallax \\
Perryman et al. 1998............ & $3.348 \pm 0.129$ & Trigonometric parallax \\
de Bruijne et al. 2001 .............. & $3.372 \pm 0.039$ & Kinematic parallax \\
\hline
\end{tabular}

(Bahcall et al. 2001). Models compatible with helioseismology that include both rotational mixing and diffusion have surface abundances of $Y_{\odot \text { surf }}=0.249 \pm 0.003$ and $Z_{\odot \text {,surf }}=$ 0.0176 , which imply an initial composition of $Y_{\odot}=0.274$ and $Z_{\odot}=0.019 \pm 0.001$ and $\alpha=1.85$. Using these values to extrapolate a model for the Hyades at $Z$ (Hyades)/ $Z_{\odot}=1.35$, we would derive $Y=0.280$ for the Hyades. These are called the "diffusion" models; these also follow the effects of microscopic diffusion, even though it does not produce significant effects at the age of the Hyades.

\section{PHOTOMETRY, MASSES, AND RADII FOR VB 22}

Table 2 summarizes three high-precision distance estimates to VB 22: from an orbital parallax (Peterson \& Solensky 1988) solution, ${ }^{2}$ the Hipparcos trigonometric parallax (Perryman et al. 1998), and the kinematic parallax (de Bruijne et al. 2001). These are all in excellent agreement.

The basic photometric data for the VB 22 system are summarized in Table 3. The first two rows of that table show the Schiller \& Malone (1987) photometry for VB 22 (i.e., both stars together) in $B, V$, and (Cousins) $I_{\mathrm{C}}$, along with the derived luminosity ratio in each filter, where the errors are taken from that paper. Following this are the apparent magnitudes in each band for the individual components derived from the luminosity ratios. Note that the photometric errors are significant for the secondary and will be accounted for in the discussion below. Finally, we derive the difference in absolute magnitude between the primary and secondary in each filter and also include the absolute magnitudes that would be obtained from the kinematic parallax (de Bruijne et al. 2001) of VB 22, again with errors in the individual components.

In Table 4 we compare the model radii and temperatures at the masses derived for each component of VB 22 by Torres \& Ribas (2002) to their solution (top ten rows of the table); we also compare the models to the earlier estimates from Peterson \& Solensky (1988; last six rows). The latter

${ }^{2}$ This value was apparently misquoted by Lebreton et al. (2001) in their Table 1 but does not affect their analysis. values were the ones used by Lebreton et al. (2001). In the comparison with the Torres \& Ribas (2002) solution, we tabulate the properties of the model both with and without diffusion, while we show only the no-diffusion case in the comparison with Peterson \& Solensky (1988). The quantities derived from the model are on the scale where the Sun has $M_{\mathrm{bol}}=4.746$ and the radii and effective temperatures are obtained from the solar-calibrated helium and mixing length.

The first thing to note is that in all cases the model radii at the observed masses are considerably different from the radii derived from the analysis of the eclipses by $2.5-4.5$ times the formal errors derived from propagating the mass error. This indicates that there is an inconsistency ${ }^{3}$ in the observational determination of mass, radius, and luminosity compared to the model. Since luminosity is determined by a combination of temperature and radius, we need to examine these separately to pinpoint the source of this inconsistency.

To quantify the size of the mismatch in temperature, we also show in Table 4 the model temperatures at the observed masses of VB 22A and VB 22B using the mass/radius relation in the models. We also compare this to the case in which we take the observed radius as correct, which would imply a larger temperature for VB 22A, where the observed radius is smaller than in the model, and a smaller temperature for VB 22B. Here the required changes in temperature are considerable, amounting to $150-190 \mathrm{~K}$.

The effect of including diffusion is shown in the top part of the table for the Torres \& Ribas (2002) solution. For the same starting helium abundance the effects of the precise value of the mixing length are very small for luminosity and modest even for the effective temperature: the diffusion models would be roughly $35 \mathrm{~K}$ hotter than the no-diffusion models for the primary and $13 \mathrm{~K}$ hotter for the secondary. Thus, the inconsistency with the models is not caused by the treatment of diffusion in the solar calibration.

Because the Hyades has been well studied spectroscopically, there exist independent estimates of the luminosity/ temperature relation. In Figure 2 we show spectroscopically derived temperatures for a subset of stars in the recent study of Hyades abundances by Paulson et al. (2003). Stars with $T<6000 \mathrm{~K}$ and good $B V I_{\mathrm{C}} K_{s}$ photometry are shown as open circles with error bars. The values of $M_{V}$ are derived

\footnotetext{
${ }^{3}$ Since $L \propto R^{2} T_{\text {eff }}^{4}$, one could match the models to the data using two of the quantities $\left(L, R, T_{\text {eff }}\right)$, but not all three simultaneously. Another indication that something is the matter is that both stars formally have the same gravity in the Torres \& Ribas (2002) analysis, which contradicts the strong prediction from theory that the mean density increases with decreasing mass for main-sequence stars. The models predict $\log g(\mathrm{VB} 22 \mathrm{~A})-\log g$ $($ VB 22B $)=-0.11$.
}

TABLE 3

Photometry of VB 22 ANd Derived Quantities

\begin{tabular}{|c|c|c|c|c|}
\hline Quantity & Star(s) & $V$ & $B$ & $I_{\mathrm{C}}$ \\
\hline Photometry.... & $\mathrm{A}+\mathrm{B}$ & $8.319 \pm 0.009$ & $9.075 \pm 0.010$ & $7.486 \pm 0.006$ \\
\hline$L_{\mathrm{A}} /\left(L_{\mathrm{A}}+L_{\mathrm{B}}\right) \ldots \ldots \ldots \ldots \ldots$ & $\ldots$ & $0.892 \pm 0.006$ & $0.928 \pm 0.004$ & $0.823 \pm 0.008$ \\
\hline \multirow[t]{2}{*}{ Photometry ......................... } & A & $8.443 \pm 0.011$ & $9.156 \pm 0.011$ & $7.697 \pm 0.012$ \\
\hline & B & $10.735 \pm 0.061$ & $11.932 \pm 0.061$ & $9.366 \pm 0.050$ \\
\hline$M_{\lambda}(\mathrm{B})-M_{\lambda}(\mathrm{A}) \ldots \ldots \ldots \ldots$ & & $2.29 \pm 0.06$ & $2.78 \pm 0.06$ & $1.67 \pm 0.05$ \\
\hline \multirow{2}{*}{ 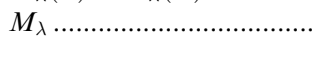 } & A & $5.07 \pm 0.035$ & $5.78 \pm 0.035$ & $4.32 \pm 0.035$ \\
\hline & B & $7.36 \pm 0.069$ & $8.56 \pm 0.069$ & $5.99 \pm 0.060$ \\
\hline
\end{tabular}


from individual kinematic parallaxes (de Bruijne et al. 2001); errors in this quantity are dominated by distance errors rather than photometric errors. Paulson et al. (2003) do not list individual temperature errors, so we took $\pm 50 \mathrm{~K}$ as a representative value, derived from Paulson et al.'s comparison of their temperatures with those in previous studies. The solid line in the figure is the Hyades isochrone derived in this paper. The filled circles show the temperature of the isochrone at the Torres \& Ribas (2002) masses. The filled triangles display the temperatures that would be found for the components of VB 22 if the measured radii were correct. The agreement between the isochrone and the spectroscopic temperatures is excellent, which shows that the luminosity/ radius relationship in the models is nearly correct, at least under the assumption that all Hyades stars have identical metallicity. If on the other hand the luminosity/temperature relation in the models were adjusted to match the radii in the Torres \& Ribas (2002) solution (VB 22A hotter by 200 $\mathrm{K}$, VB 22B cooler), then there would be a difference of about 0.15 dex between the hottest and coolest stars in the Paulsen et al. sample (the hotter stars would come out more metal-rich).

In Figure 3 we compare the isochrones to the Torres \& Ribas (2002) solution in $M_{V}, B-V$, and $V-I_{\mathrm{C}}$ as a function of mass. The isochrone is slightly brighter than the data, which indicates that the distance to VB 22 is underestimated or, as we discuss in $\S 4$, that the helium abundance we adopted for the Hyades is too high. The color-temperature relation in the isochrone differs from the inferred colors of the binary components, but in this paper we are mainly concerned with comparing the model luminosities to the data.

Table 5 summarizes the errors in absolute magnitude, radius, or effective temperature that are contributed by different effects. To compute the result of the uncertainty in mass, we assume the Torres \& Ribas (2002) error of 0.0062

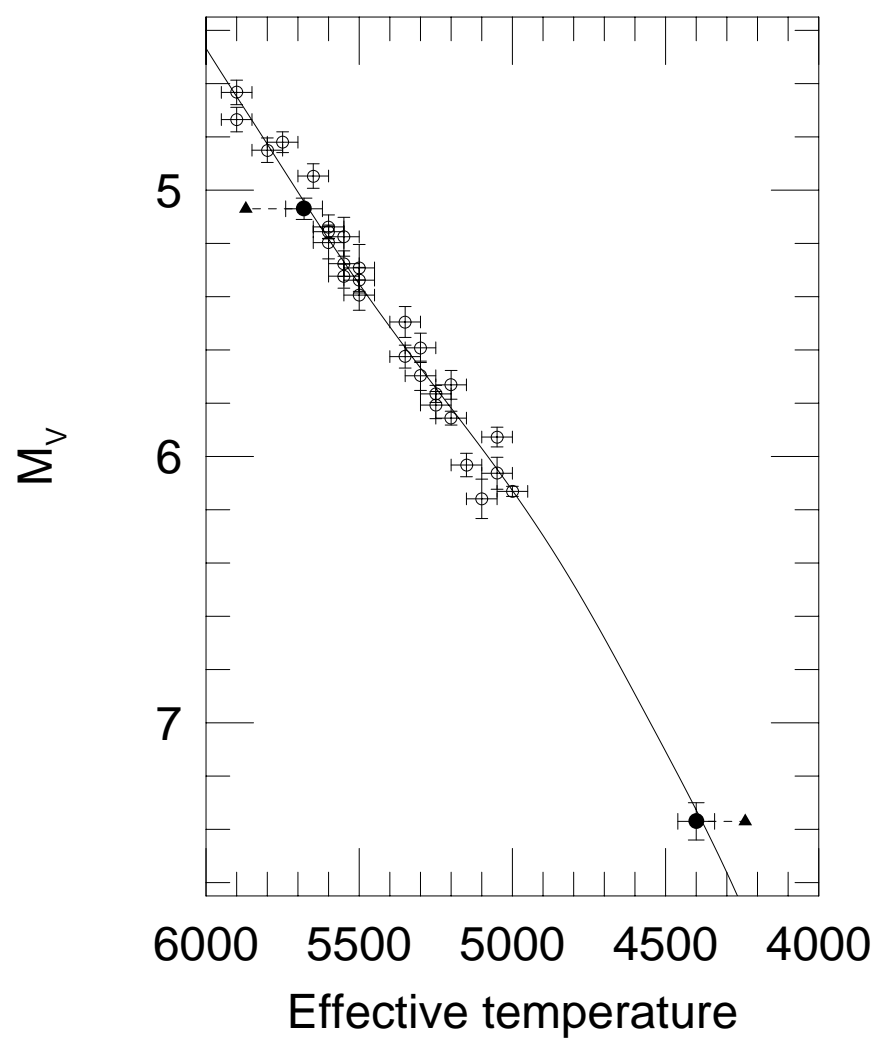

FIG. 2.-Comparison of isochrone and spectroscopic temperature scales for the Hyades. The solid line is the theoretical isochrone in Table 1, while the open points with error bars show spectroscopic temperatures from Paulson et al. (2003), along with absolute visual magnitude computed from Hyades kinematic parallaxes (de Bruijne et al. 2001). The filled circles are for VB $22 \mathrm{~A}$ and VB 22B at the isochrone temperature and measured masses for each component, while the triangles indicate the temperatures that would be derived by forcing the models to have the radius indicated by the Torres \& Ribas (2002) solution.

TABLE 4

Physical Data For VB 22 and Models

\begin{tabular}{|c|c|c|c|}
\hline Quantity & VB 22A & VB 22B & Comment \\
\hline \multicolumn{4}{|c|}{ Torres \& Ribas 2002} \\
\hline$M / M_{\odot}($ observed $) \ldots \ldots \ldots \ldots \ldots$ & $1.0591 \pm 0.0062$ & $0.7605 \pm 0.0062$ & TR02 solution \\
\hline 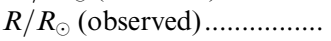 & $0.900 \pm 0.016$ & $0.768 \pm 0.010$ & TR02 solution \\
\hline \multirow[t]{2}{*}{$R / R_{\odot}($ model $) \ldots \ldots \ldots \ldots \ldots \ldots \ldots$} & $0.962 \pm 0.007$ & $0.714 \pm 0.007$ & No diffusion \\
\hline & $0.955 \pm 0.007$ & $0.712 \pm 0.007$ & With diffusion \\
\hline \multirow{4}{*}{$T_{\text {eff }}($ model $) \ldots \ldots \ldots \ldots \ldots \ldots \ldots \ldots \ldots$} & $5680 \pm 60$ & $4400 \pm 60$ & No diffusion, at listed mass \\
\hline & $5870 \pm 50$ & $4240 \pm 30$ & No diffusion, if observed radius is correct \\
\hline & $5780 \pm 60$ & $4460 \pm 60$ & With diffusion, at listed mass \\
\hline & $5950 \pm 60$ & $4290 \pm 30$ & With diffusion, if TR 02 radius is correct \\
\hline \multirow{2}{*}{$M_{\mathrm{bol}}($ model $) \ldots \ldots \ldots \ldots \ldots \ldots \ldots \ldots$} & 4.90 & 6.66 & No diffusion \\
\hline & 4.83 & 6.59 & With diffusion \\
\hline \multicolumn{4}{|c|}{ Peterson \& Solensky 1988} \\
\hline$M / M_{\odot}($ observed $) \ldots \ldots \ldots \ldots \ldots$ & $1.072 \pm 0.062$ & $0.769 \pm 0.005$ & PS88 solution \\
\hline$R / R_{\odot}$ (observed) $\ldots \ldots \ldots \ldots \ldots \ldots$ & $0.905 \pm 0.029$ & $0.773 \pm 0.015$ & PS88 solution \\
\hline 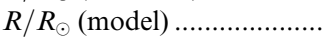 & $0.974 \pm 0.007$ & $0.714 \pm 0.007$ & \\
\hline$M_{\mathrm{bol}}($ model $) \ldots$ & 4.84 & 6.61 & \\
\hline \multirow{2}{*}{ 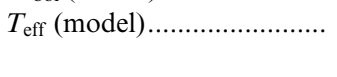 } & $5930 \pm 50$ & $4290 \pm 40$ & If $\mathrm{PS} 88$ radius is correct \\
\hline & $5720 \pm 60$ & $4400 \pm 60$ & At listed mass \\
\hline
\end{tabular}

Notes._PS88 $=$ Peterson $\&$ Solensky 1988. TR02 $=$ Torres $\&$ Ribas 2002. All the comparisons with PS88 assume no diffusion. 


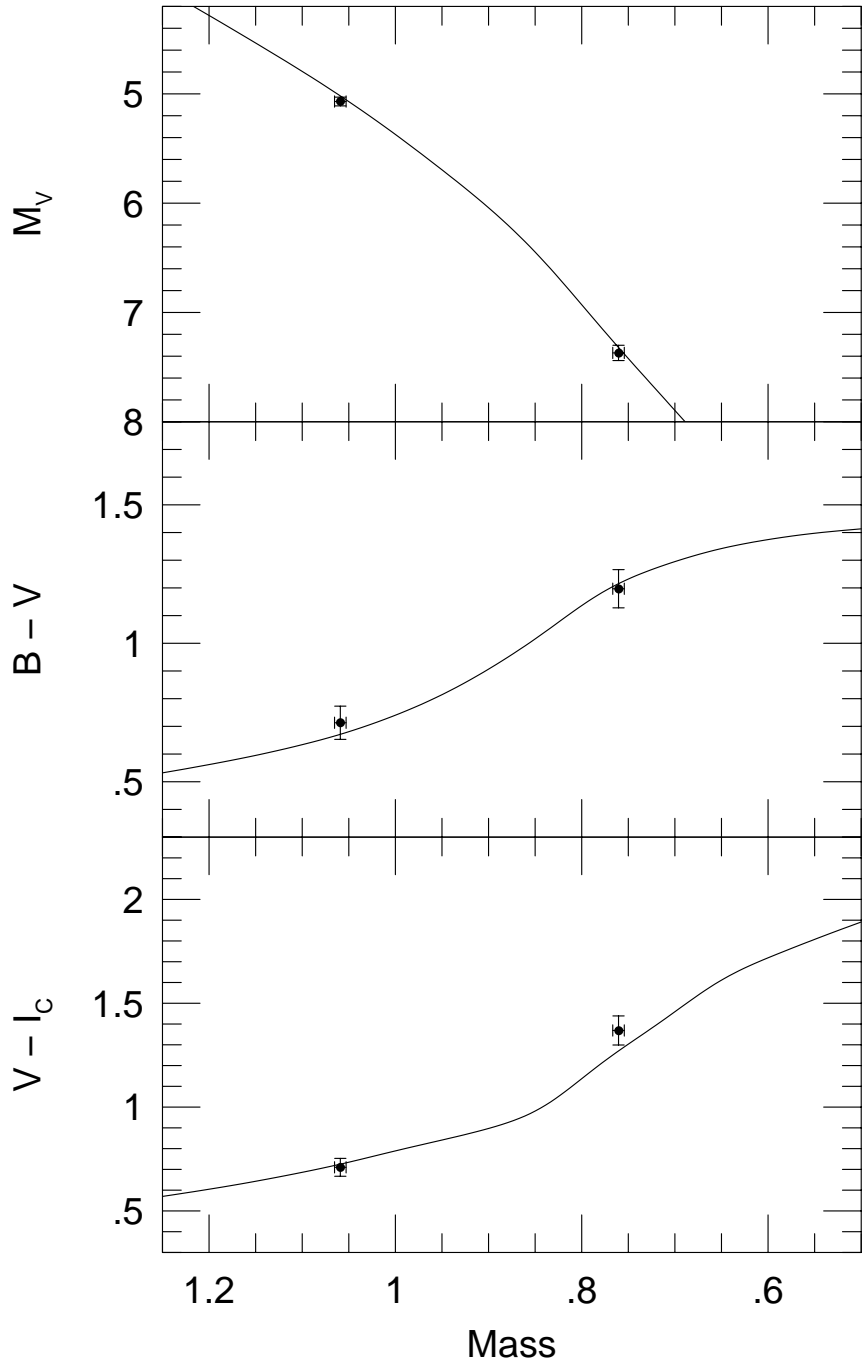

FIG. 3.-Comparison of the isochrone to data for VB 22. The isochrone is shown as a solid line, and the values for VB 22A and VB 22B are shown as points with error bars.

$M_{\odot}$ for each star. The errors from $[\mathrm{Fe} / \mathrm{H}]$ assume $\sigma[\mathrm{Fe} / \mathrm{H}]$ $=0.05$ dex per star, while those listed for the bolometric correction were computed by taking the largest difference between the inferred fluxes for that filter between three different color calibrations (below) and dividing by 2. We have also computed errors that would result if the metal abundance were known with vanishingly small errors; these are shown in the rows labeled "no $Z$." Because the sign of changes in metallicity is the same for both components, the errors in their relative fluxes are smaller; we give these values in the row labeled " B - A."

We compare the absolute magnitudes of the two components of VB 22 to the models in Table 6, where we employ models lacking diffusion but with two alternative color calibrations, those of Allard \& Hauschildt (1995) and Alonso, Arribas, \& Martínez-Roger (1996), in addition to the one employed as our base case (Lejeune et al. 1998). The comparison is done at fixed mass. In general, the different color calibrations change the luminosity by only a few hundredths of a magnitude. We take the scatter in the luminosities indicating the size of errors in the bolometric corrections; these are shown in Table 5 . We show the effects of including diffusion or of forcing the temperature scale to match the observed stellar radii in Table 7. These models are for the Lejeune et al. (1998) color calibration only.

The agreement between theory and observation is impressive for the no-diffusion models and well within the expected errors for both the absolute luminosities and the relative luminosities. Overall, the different color calibrations agree best for the $V$ and $B$ bands, while there is more scatter in the predicted $I_{\mathrm{C}}$-band luminosities. The relative fluxes are very close to the predicted level for the $V$ and $B$ bands and are mildly inconsistent with the $I_{\mathrm{C}}$-band fluxes, especially for VB 22B, where the models are fainter than the data by 0.1 mag or so. This comparison indicates that the problem is most likely in the $I_{\mathrm{C}}$-band bolometric corrections rather than in the $V$-band bolometric corrections.

However, the agreement is not preserved if the effective temperatures are altered to the values inferred from the eclipse data. Essentially, choosing a lower effective temperature for the secondary drives down the $V$ - and $B$-band fluxes while slightly increasing the $I$-band flux. As a result, the relative flux differences in the $V$ and $B$ bands become much larger. This result is insensitive to the metallicity of the Hyades because decreases in the metallicity affect the luminosity and effective temperature of both components in the same sense, while the model radii are insensitive to the metallicity. We therefore conclude that the relative fluxes in different bands, the mass-luminosity relationship, and the radii obtained from the eclipse data are not consistent with one another. One of the three must be in error. Because of the insensitivity of the mass-luminosity relationship to errors in the input physics, we view it as more likely that there is some unresolved issue in one of the two other ingredients. As in previous analyses of this system, we note that there is no obvious single change in the input physics that can reconcile the models with both components simultaneously. What we have added is evidence that these radii are also inconsistent with the flux ratios.

TABLE 5

ERror ANALYSIS

\begin{tabular}{|c|c|c|c|c|c|c|c|c|c|c|}
\hline \multirow[b]{2}{*}{ TheORETICAL ERrors } & \multicolumn{5}{|c|}{ Star A } & \multicolumn{5}{|c|}{ Star B } \\
\hline & $V$ & $B$ & $I_{\mathrm{C}}$ & $R / R_{\odot}$ & $T_{\text {eff }}$ & $V$ & $B$ & $I_{\mathrm{C}}$ & $R / R_{\odot}$ & $T_{\text {eff }}$ \\
\hline$\sigma$ (mass). & 0.03 & 0.03 & 0.04 & 0.006 & 18 & 0.06 & 0.07 & 0.04 & 0.006 & 27 \\
\hline$\sigma([\mathrm{Fe} / \mathrm{H}]) \ldots \ldots \ldots \ldots \ldots \ldots$ & 0.06 & 0.08 & 0.04 & 0.003 & 58 & 0.09 & 0.12 & 0.06 & 0.003 & 56 \\
\hline$\sigma(\mathrm{BC}) \ldots \ldots \ldots \ldots \ldots \ldots \ldots \ldots \ldots \ldots \ldots \ldots \ldots$ & 0.02 & 0.03 & 0.03 & . & . & 0.03 & 0.01 & 0.04 & & 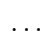 \\
\hline$\sigma($ total $) \ldots \ldots \ldots \ldots \ldots \ldots \ldots$ & 0.07 & 0.09 & 0.06 & 0.007 & 61 & 0.11 & 0.14 & 0.08 & 0.007 & 62 \\
\hline$\sigma($ total $)($ no $Z) \ldots \ldots \ldots \ldots . .$. & 0.04 & 0.04 & 0.05 & 0.006 & 18 & 0.07 & 0.07 & 0.06 & 0.007 & 62 \\
\hline$\sigma($ total $)(\mathrm{B}-\mathrm{A}) \ldots \ldots \ldots \ldots$ & 0.08 & 0.09 & 0.08 & $\ldots$ & $\ldots$ & ... & $\ldots$ & $\ldots$ & $\ldots$ & $\ldots$ \\
\hline
\end{tabular}


TABLE 6

EfFect of Color Calibration on No-Diffusion Model

\begin{tabular}{|c|c|c|c|c|c|c|c|}
\hline \multirow[b]{2}{*}{ QUANTITY } & \multicolumn{3}{|c|}{ COLOR CALIBRATION } & \multirow[b]{2}{*}{ DATA } & \multicolumn{3}{|c|}{ DifFERENCE (Model - Data) } \\
\hline & $\mathrm{A} \& \mathrm{H}^{\mathrm{a}}$ & $\mathrm{AAM}^{\mathrm{b}}$ & $\mathrm{LCB}^{\mathrm{c}}$ & & $\mathrm{A} \& \mathrm{H}^{\mathrm{a}}$ & $\mathrm{AAM}^{\mathrm{b}}$ & $\mathrm{LCB}^{\mathrm{c}}$ \\
\hline \multicolumn{8}{|c|}{ VB 22A } \\
\hline$M_{V} \ldots \ldots \ldots \ldots \ldots \ldots \ldots \ldots$ & 5.02 & 5.05 & 5.03 & $5.07 \pm 0.04$ & -0.05 & -0.02 & -0.04 \\
\hline$M_{B} \ldots \ldots \ldots \ldots \ldots \ldots \ldots \ldots$ & 5.73 & 5.71 & 5.71 & $5.78 \pm 0.04$ & -0.05 & -0.07 & -0.02 \\
\hline$M_{I}$ & 4.29 & 4.36 & 4.30 & $4.32 \pm 0.04$ & -0.03 & +0.04 & -0.02 \\
\hline$B-V \ldots$ & 0.71 & 0.66 & 0.68 & $0.71 \pm 0.06$ & $\ldots$ & $\ldots$ & $\cdots$ \\
\hline$V-I \ldots$ & 0.73 & 0.69 & 0.73 & $0.75 \pm 0.06$ & $\cdots$ & . & \\
\hline \multicolumn{8}{|c|}{ VB 22B } \\
\hline$M_{V} \ldots \ldots \ldots \ldots \ldots \ldots \ldots \ldots$ & 7.37 & 7.38 & 7.34 & $7.36 \pm 0.07$ & +0.01 & +0.02 & -0.02 \\
\hline$M_{B} \ldots \ldots \ldots \ldots \ldots \ldots \ldots \ldots$ & 8.53 & 8.52 & 8.53 & $8.56 \pm 0.07$ & -0.03 & -0.04 & -0.03 \\
\hline$M_{I} \ldots \ldots \ldots \ldots \ldots \ldots \ldots \ldots$ & 6.09 & 6.15 & 6.10 & $5.99 \pm 0.06$ & +0.10 & +0.16 & +0.11 \\
\hline$B-V \ldots \ldots \ldots \ldots \ldots \ldots \ldots$ & 1.20 & 1.14 & 1.19 & $1.20 \pm 0.07$ & $\ldots$ & $\ldots$ & $\ldots$ \\
\hline$V-I \ldots \ldots \ldots \ldots \ldots \ldots \ldots$ & 1.37 & 1.23 & 1.24 & $1.37 \pm 0.07$ & $\ldots$ & $\ldots$ & $\ldots$ \\
\hline
\end{tabular}

a Allard \& Hauschildt 1995

b Alonso et al. 1996.

${ }^{c}$ Lejeune et al. 1998.

\section{THE HELIUM ABUNDANCE OF THE HYADES}

Because the luminosity of stellar models at fixed mass is very sensitive to the helium abundance $\left(\partial M_{\mathrm{bol}} / \partial Y=-10\right)$, we can formally derive an initial helium abundance for the Hyades: using the de Bruijne et al. (2001) distance to VB 22, we find $Y=0.271 \pm 0.006$ for the no-diffusion models. The models including diffusion are brighter than those without, indicating that the initial choice of $Y=0.280$ was too high at the assumed distance. Correcting those models brings the estimated helium abundance down to $Y=0.271$, showing that the derived helium abundance is nearly independent of the details of the solar calibration.

If we adopt a primordial helium abundance of 0.245 , the Hyades would give a slope $\Delta Y / \Delta Z=1.11 \pm 0.25$, smaller than the values of 1.5 and 1.2 obtained from solar models with and without diffusion, respectively. If the more realistic initial solar helium abundance (including diffusion) and the Hyades helium abundance are taken at face value, they sug- gest a scatter in helium at fixed metal abundance of order 0.009 ; however, this range is only marginally significant. If we take this as a $1 \sigma$ error range, it would imply only a small resulting error in a cluster distance modulus at fixed $[\mathrm{Fe} / \mathrm{H}]$ of order $0.027 \mathrm{mag}$.

The Hyades helium abundance we derive is equal within the errors to the solar value $Y=0.273$ in models excluding diffusion. The solar models with diffusion would predict a higher helium abundance of 0.280 , which is not favored by the data; however, it is only of order $2 \sigma$ from the measurement. Isochrones are less sensitive to changes in helium than models of a given mass, with a change of 0.01 in helium causing a change in $M_{V}$ at fixed $T_{\text {eff }}$ of 0.03 . We can include this in our next paper in the error budget for the absolute distances.

This value is higher than that obtained by Lebreton et al. (2001), who found $Y=0.255 \pm 0.009$. There are two reasons for this difference: First, we adopted the revised masses of Torres \& Ribas (2002); these yield predicted

TABLE 7

Alternative Models

\begin{tabular}{|c|c|c|c|c|}
\hline \multirow[b]{2}{*}{ QUANTITY } & \multicolumn{2}{|c|}{ Alternative Model $^{\mathrm{a}}$} & \multicolumn{2}{|c|}{ DiFFERENCE (MODEL - DATA) } \\
\hline & Diffusion & Force Radius & Diffusion & Force Radius \\
\hline \multicolumn{5}{|c|}{ VB 22A } \\
\hline$M_{V} \ldots \ldots \ldots \ldots \ldots \ldots \ldots$ & 5.00 & 4.97 & -0.07 & -0.10 \\
\hline$M_{B} \ldots \ldots \ldots \ldots \ldots \ldots \ldots \ldots$ & 5.65 & 5.62 & -0.13 & -0.16 \\
\hline$M_{I}$ & 4.34 & 4.28 & +0.02 & -0.04 \\
\hline B-V & 0.65 & 0.65 & $\ldots$ & $\ldots$ \\
\hline$V-I \ldots \ldots \ldots \ldots \ldots \ldots \ldots \ldots$ & 0.66 & 0.69 & $\ldots$ & $\ldots$ \\
\hline \multicolumn{5}{|c|}{ VB 22B } \\
\hline$M_{V} \ldots \ldots \ldots \ldots \ldots \ldots \ldots$ & 7.48 & 7.31 & +0.12 & -0.05 \\
\hline$M_{B} \ldots \ldots \ldots \ldots \ldots \ldots \ldots \ldots \ldots \ldots \ldots \ldots$ & 8.73 & 8.47 & -0.17 & -0.09 \\
\hline$M_{I} \ldots \ldots \ldots \ldots \ldots \ldots \ldots \ldots$ & 6.14 & 5.92 & +0.22 & +0.15 \\
\hline$B-V \ldots \ldots \ldots \ldots \ldots \ldots \ldots$ & 1.25 & 1.16 & $\ldots$ & $\ldots$ \\
\hline$V-I \ldots \ldots \ldots \ldots \ldots \ldots \ldots$ & 1.34 & 1.39 & $\ldots$ & $\ldots$ \\
\hline
\end{tabular}

a Lejeune et al. 1998 color calibration. 
luminosities that are 0.08-0.09 mag fainter than found by Peterson \& Solensky (1988), resulting in a higher helium abundance. Second, our models employ a number of ingredients not used by Lebreton et al.; as they note, using the OPAL equation of state, Kurucz model atmospheres rather than a gray atmosphere, and a higher mixing length all increase the inferred value of $Y$.

The mixture of heavy elements can also have an impact on the properties of the models. There have been two relatively recent revisions of the Grevesse \& Noels (1993) abundance scale for the Sun that has been used in this paper. Grevesse \& Sauval (1998) have reduced the CNO abundances, while Asplund (2000) has proposed a downward revision of $9.2 \%$ in the zero point between the meteoritic and photospheric abundance scales. This would not alter those abundances not tied to the meteoritic scale $(\mathrm{C}, \mathrm{N}, \mathrm{O}, \mathrm{Ne}$, etc.) but would affect important interior opacity sources such as $\mathrm{Si}$ and Fe. Helioseismic tests (Bahcall et al. 2001) indicate that the Grevesse \& Sauval (1998) mixture marginally degrades the agreement with the measured solar convection zone depth, but by a degree that is consistent with other known theoretical uncertainties. The solar helium abundance is insensitive to the $\mathrm{CNO}$ abundances. The Asplund (2000) mixture has a small impact on the solar sound speed, but the lower iron abundance results in a lower solar helium abundance. This is in disagreement with the measured surface abundance of helium at the 2-3 $\sigma$ level; Bahcall et al. (2001) were not able to rule this out because the degree of disagreement is sensitive to systematic errors in the helium abundance determination arising from the equation of state.

We have verified that adopting the Grevesse \& Sauval (1998) mixture instead of the Grevesse \& Noels (1993) mixture produces only small changes in the isochrones (or even in the more sensitive tests possible in VB 22). The Asplund (2000) mixture would imply a Hyades helium abundance (including diffusion) that is comparable to the Grevesse \& Noels (1993) or Grevesse \& Sauval (1998) helium abundance inferred in the absence of diffusion. We have also explored the effects of including small deviations from the solar mix in the Hyades as measured by Paulson et al. (2003) and found that the impact would be of the same order as the difference between the choice of the solar element mix.

\section{SUMMARY AND REMARKS}

In this paper we have begun a new analysis of open cluster distances by performing stringent tests of the luminosity/ temperature relation for new isochrones with updated physics. We have explored the effect of varying many details of the models, including the abundance mix of helium and metals, and concluded that the models match the relative temperatures and luminosities of the components of the binary VB 22 in the Hyades. Along the way, we have derived a helium abundance that is not much different from that in the Sun, even though the Hyades is more metal-rich.

The resulting helium abundance is almost insensitive to the details of the solar calibration used to generate the isochrone. Models using a solar calibration consistent with helioseismology predict a high value of $Y$ for the Hyades; the resulting isochrone, however, is too bright at fixed mass, which leads to the conclusion that the Hyades helium abundance is not much different from the Sun's initial value of $Y=0.272$. This may imply a scatter in the helium abundance of about $\delta Y=0.01$ at fixed metallicity in the solar neighborhood. While this would produce a real effect on the luminosity of the isochrones at fixed mass, we have argued that the effect on the isochrones at fixed color would be much smaller and would not produce big errors in the distance estimates derived from main-sequence fitting.

The principal result of this paper is our demonstration that there is an inconsistency between the solar models and the binary data in mass, luminosity, temperature, and radius. External checks on the abundance and temperature scales from recent high-precision abundances in the Hyades (Paulson et al. 2003) indicate that the problem lies with the observational determination of the radii. We conclude from this that we can adjust the isochrones to match the Hyades photometry by leaving the stellar luminosity and temperatures as they are and computing corrections to the color/ temperature relations; this is the subject of our next paper.

The comparison between the model colors and absolute magnitude and the data for VB 22 may be complicated by the presence of spots on the stellar surfaces. In the Pleiades, stars with the luminosities of VB 22B fall up to $0.5 \mathrm{mag}$ below the main sequence in $V$ and $B-V$ as defined by the Hyades (van Leeuwen et al. 1987; Stauffer et al. 2003); the difference is negligible when $V$ and $V-I$ are used. Stauffer et al. (2003) attribute this to the presence of hot and cool regions on the surfaces of the rapidly rotating Pleiades stars as compared with their slowly rotating counterparts in the Hyades. The orbital period of VB 22 is 5.6 days (Schiller \& Malone 1987). If both members of VB 22 were corotating, this would correspond to equatorial rotation speeds of only $8 \mathrm{~km} \mathrm{~s}^{-1}$ for VB 22A and $6 \mathrm{~km} \mathrm{~s}^{-1}$ for VB 22B (the binaries are well detached). These values are not much different from the average $v \sin i$ for Hyades stars of similar colors in Paulson et al. (2003) and considerably slower than the projected rotation speed $(v \sin i)$ for the Pleiades stars with the most anomalous $B-V$ color. The VB 22 system, however, has exhibited flares and color variations outside eclipses, so active regions may indeed be important at some level (Schiller \& Malone 1987).

The work reported here was supported in part by the National Science Foundation under grants AST 97-31621 and AST 02-06008 to the Ohio State University Research Foundation. We wish to thank the anonymous referee for many helpful comments.
Alexander, D. R., \& Ferguson, J. W. 1994, ApJ, 437, 879

Allard, F. \& Hauschildt, P. H. 1995, ApJ, 445, 433

Alonso, A., Arribas, S., \& Martínez-Roger, C. 1996, A\&A, 313, 873 Asplund, M. 2000, A\&A, 359, 755

Bahcall, J. N., Pinsonneault, M. H., \& Basu, S. 2001, ApJ, 555, 990

Boesgaard, A. M., \& Friel, E. D. 1990, ApJ, 351, 467

Böhm-Vitense, E. 1958, Z. Astrophys., 46, 108

Bono, G., Balbi, A., Cassisi, S., Vittorio, N., \& Buonanno, R. 2002, ApJ, 568,463

\section{REFERENCES}

Castellani, V., Degl'Innocenti, S., \& Prada Moroni, P. G. 2001, MNRAS, 320,66

Cox, J. P., \& Guili, R. T. 1968, Principles of Stellar Structure (New York: Gordon \& Breach)

de Bruijne, J. H. J., Hoogerwerf, R., \& de Zeeuw, P. T. 2001, A\&A, 367, 111

Grevesse, N., \& Noels, A. 1993, in Origin and Evolution of the Elements, ed. M. Prantzos, E. Vangioni-Flam, \& M. Cassé (Cambridge: Cambridge Univ. Press), 15 
Grevesse, N., \& Sauval, A. J. 1998, Space Sci. Rev., 85, 161

Gruzinov, A., \& Bahcall, J. 1998, ApJ, 504, 996

Guenther, D. B., Demarque, P., Kim, Y.-C., \& Pinsonneault, M. H. 1992, ApJ, 387, 372

Iglesias, C. A., \& Rogers, F. J. 1996, ApJ, 464, 943

Jimenez, R., Flynn, C., MacDonald, J., \& Gibson, B. K. 2003, Science, 299, 1552

Lastennet, E., Valls-Gabaud, D., Lejeune, T., \& Oblak, E. 1999, A\&A, 349, 485

Lebreton, Y., Fernandes, J., \& Lejeune, T. 2001, A\&A, 374, 540

Lejeune, T., Cuisinier, F., \& Buser, R. 1998, A\&AS, 130, 65

Makarov, V. V. 2002, AJ, 124, 3299

McClure, R. D. 1982, ApJ, 254, 606

Paulson, D. B., Sneden, C., \& Cochran, W. D. 2003, AJ, 125, 3185

Percival, S. M., Salaris, M., \& Kilkenny, D. 2003, A\&A, 400, 541

Perryman, M. A. C., et al. 1998, A\&A, 331, 81

Peterson, D. M., \& Solensky, R. 1988, ApJ, 333, 256
Radick, R. R., Thompson, D. T., Lockwood, G. W., Duncan, D. K., \& Baggett, W. E. 1987, ApJ, 321, 459

Robichon, N., Arenou, F., Mermilliod, J.-C., \& Turon, C. 1999, A\&A, 345, 471

Rogers, F. J., Swenson, F. J., \& Iglesias, C. A. 1996, ApJ, 456, 902

Saumon, D., Chabrier, G., \& Van Horn, H. M. 1995, ApJS, 99, 713

Schiller, S. J., \& Malone, E. F. 1987, AJ, 93, 1471

Sills, A., Pinsonneault, M. H., \& Terndrup, D. M. 2000, ApJ, 534, 335

Stauffer, J. R., Jones, B. F., Backman, D., Hartmann, L. W., Barrado y Navascués, D., Pinsonneault, M. H., Terndrup, D. M., \& Muench, A. 2003, AJ, 126, 833

Terndrup, D. M., Stauffer, J. R., Sills, A., Yuan, Y., Jones, B. F., Fischer, D., \& Krishnamurthi, A. 2000, AJ, 119, 1303

Thuan, T. X., \& Izotov, Y. I. 2002, Space Sci. Rev., 100, 263

Torres, G., \& Ribas, I. 2002, ApJ, 567, 1140

van Leeuwen, F. 1999, A\&A, 341, L71

van Leeuwen, F., Alphenaar, P., \& Meys, J. J. M. 1987, A\&AS, 67, 483 